\title{
Formation mechanism of spheroidal carbide in ultra-low carbon ductile cast iron
}

\author{
Bin-guo Fu, Zhuo-qing Li, Xue-bo Zhao, Zhen Shen, Guo-lu Li, and *Jin-hai Liu \\ School of Materials Science and Engineering, Hebei University of Technology, Tianjin 300130, China
}

\begin{abstract}
The formation mechanism of the spheroidal carbide in the ultra-low carbon ductile cast iron fabricated by the metal mold casting technique was systematically investigated. The results demonstrated that the spheroidal carbide belonged to eutectic carbide and crystallized in the isolated eutectic liquid phase area. The formation process of the spheroidal carbide was related to the contact and the intersection between the primary dendrite and the secondary dendrite of austenite. The oxides of magnesium, rare earths and other elements can act as heterogeneous nucleation sites for the spheroidal carbide. It was also found that the amount of the spheroidal carbide would increase with an increase in carbon content. The cooling rate has an important influence on the spheroidal carbide under the same chemical composition condition.
\end{abstract}

Key words: ultra-low carbon ductile cast iron; cooling rate; nucleation core; spheroidal carbides; formation mechanism

CLC numbers: TG143.5 Document code: A Article ID: 1672-6421(2016)05-346-06

\begin{abstract}
A ustempered ductile iron (ADI) as a kind of very promising engineering material, is extensively used in gears, heavy machinery and transportation industries due to the combination of superior properties, such as high strength with good ductility, good wear resistance and fatigue tolerance ${ }^{[1-4]}$. The microstructure of the ADI consists of a certain number of graphite nodules, and the coarse graphite nodules with a strong fragmentation effect on the matrix structures seriously damage the properties of the materials ${ }^{[5,6]}$. Therefore, people try to obtain tiny, round and uniformly distributed graphite nodules to improve the mechanical properties by reducing the carbon content, and extensive efforts have been devoted to investigating ultra-low carbon $\mathrm{ADI}^{[7]}$. The ultra-low carbon ADI can be prepared with a certain composition of ductile iron through austempering heat treatmen ${ }^{[8]}$, so an important step in the development of the ultra-low carbon ADI involves melting and casting of ultra-low carbon ductile irons. In the traditional Fe-C binary phase diagram, the right to the point (2.1wt.\%) is called iron, and the left is called steel. However, the
\end{abstract}

\footnotetext{
*Jin-hai Liu
}

Male, born in 1957, Ph.D., Professor. His research interests mainly focus on the new type of abrasive materials such as ADI, CADI and their forming technology, the development of the iron melt quality online measurement and control system.

E-mail: jhliu57@126.com

Received: 2016-04-05; Accepted: 2016-06-20 ultra-low carbon ductile iron containing low carbon content and high silicon content belongs to Fe-C-Si series alloy. Si can make the point of the maximum carbon content (point $\mathrm{E}$ in $\mathrm{Fe}-\mathrm{C}$ diagram) in austenite shift left during the non-equilibrium crystallization process $^{[9,10]}$. So eutectic graphite structure could be obtained in low carbon high silicon alloy, and the FeC-Si alloy can be called ultra-low carbon ductile iron. However, some other investigators regard this alloy should be called graphite steel ${ }^{[11]}$.

It has been reported that spheroidal carbide can further improve wear resistance and not reduce the impact toughness of the ductile irons ${ }^{[12-14]}$. However, little information is available in the literature on the carbide of the ultra-low carbon ductile iron, and the spheroidal carbide in the ultra-low carbon ductile iron has not been reported. Thus, in this present study, the ultra-low carbon ductile cast iron was fabricated by the metal mold casting technique, and the effect of the carbon content and the cooling rate on the spheroidal carbide formation have been systematically investigated. The corresponding formation mechanism was also discussed.

\section{Experimental procedure}

The cast ultra-low carbon ductile irons were obtained as cylinder blocks with chemical compositions (wt.\%): 0.7-1.2 C, 2.5-2.7 Si, 2.3-2.7 Mn, 0.3-0.5 Cr, 0.2- 
$0.4 \mathrm{Mo}, \mathrm{P}<0.03, \mathrm{~S}<0.03$ and balance of Fe. Steel scraps, iron, carbon additive, ferrosilicon, ferromanganese, low carbon ferrochromium, ferromolybdenum were used as raw materials. The melting was carried out in a medium frequency induction furnace with a holding capacity of $120 \mathrm{~kg}$. After melt was superheated up to $1,650{ }^{\circ} \mathrm{C}$ and poured to the casting ladle, the inoculation, modification and spheroidizing treatments were carried out using the sandwich method. The SC agent, silicon barium, and rare earth magnesium alloys were selected for modifier, inoculant and nodulizer, respectively. After the melt treatments finished, the alloy melt was poured into a metal mold with the dimension of $\Phi 120 \mathrm{~mm} \times 170 \mathrm{~mm}$.

For microstructural analysis of the samples with different carbon contents $(0.79 \mathrm{wt} . \%, 0.97 \mathrm{wt} . \%$ and $1.20 \mathrm{wt} . \%)$, strips of $30 \mathrm{~mm}$ long, $10 \mathrm{~mm}$ wide and $2 \mathrm{~mm}$ thick at the same location were cut from the bottom of three ingots with $0.79 \mathrm{wt} . \%$, $0.97 \mathrm{wt} . \%$ and $1.20 \mathrm{wt} . \%$ carbon content by electric discharging. Samples with different cooling rates for microstructural observation were cut from the radial-transverse planes of the $1.20 \mathrm{wt} . \%$ carbon composition ingot, and the positions of the samples were in the center ( $\mathrm{R} 0-20 \mathrm{~mm}), 1 / 2$ radius ( $\mathrm{R} 20-$ $40 \mathrm{~mm}$ ), and edges (R40-60 mm). The microstructures were observed by optical microscopy (OM; Nikon eclipse MA 100) and scanning electron microscope (SEM; Philips XL30) equipped with an energy dispersive spectroscope (EDS). Samples for OM and SEM observations were prepared according to conventional metallographic techniques. The black and white metallographic samples were etched with a solution containing 4vol.\% $\mathrm{HNO}_{3}$ and 96vol.\% alcohol and the color metallographic samples were etched with a special solution containing $0.9 \mathrm{~g}$ picric acid, $12.5 \mathrm{~g}$ caustic soda and $50 \mathrm{ml}$ distilled water for $12 \mathrm{~min}$ at the temperature of $90 \pm 1{ }^{\circ} \mathrm{C}$. The Solidcast software from the FSI Company in Unites States was used to simulate the casting process.

\section{Results and discussion}

\subsection{Distribution of spheroidal graphite and carbide}

Figure 1 shows the spheroidal graphite and carbide in the bottom of the sample with $1.20 \mathrm{wt} . \%$ carbon. It can be seen that there are many fine spheroidal graphites or particles distributed in the matrix, as shown in Fig. 1(a). After the metallographic etching, a large number of spheroidal carbides can be found in the pearlite matrix as shown in Fig. 1(b). This shows that two kinds of eutectic reaction may occur during the solidification process of the ultra-low carbon ductile iron. The formation of graphites is followed by an equilibrium crystallization process, and the formation of spheroidal carbides is followed by a non-equilibrium crystallization process due to strong chilling capacity of the metal mold.

The black and white and color metallographic microstructures of the sample with 1.20wt.\% carbon are shown in Fig. 2. It can
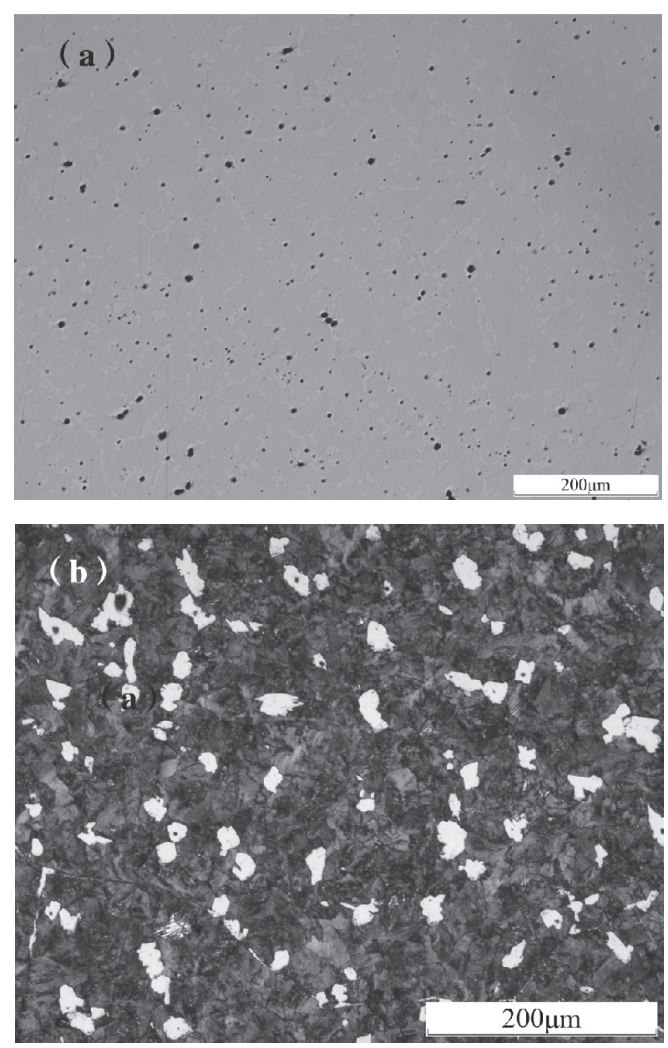

Fig. 1: Spheroidal graphites and carbides in the sample with 1.20 wt. $\%$ carbon content: (a) spheroidal graphites; (b) spheroidal carbides

be seen that the distribution of spheroidal carbides is consistent and local carbides may have certain orientation relationships with the matrix. The actual distribution position of carbide in
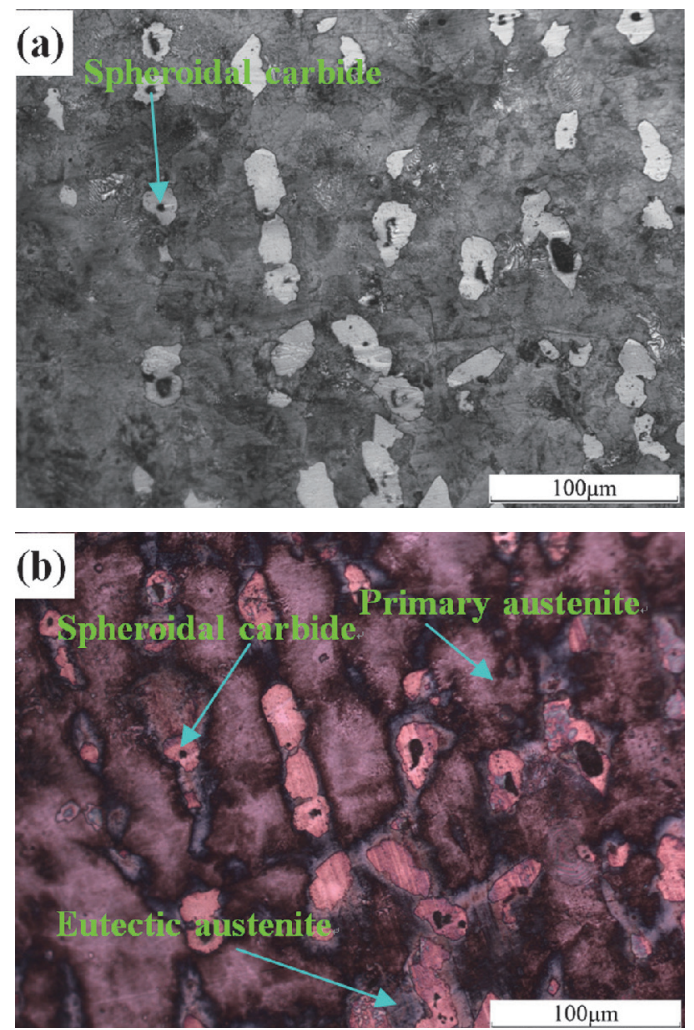

Fig. 2: OM microstructures of sample with $1.20 \mathrm{wt} . \%$ carbon composition (a) black and white; and (b) color metallographic techniques 
Fig. 2(a) is not known. However, Fig. 2(b) exhibits the light orange spheroidal carbide distributed between the gray orange primary austenites and there are a deep gray and purple regions (eutectic austenite) between the spheroidal carbide and the austenite dendrite. The spheroidal carbide can be confirmed to be eutectic carbide according to the experimental results.

Because the distribution coefficients of the alloying elements in liquid and solid phases are different during the crystallization process, microsegregation of the alloying elements present in the different phases after the non-equilibrium solidification is possible. For this experiment, the distribution of negative segregation element $\mathrm{Si}$ in the primary austenite was more than that in the eutectic austenite. The silicon content in the core was higher than that in the grain boundaries for the same grain. The distributions of Si made the degree of corrosion of the alloy matrix microstructures different after treatment by the picric acid with alkaline conditions. Thus we can determine the phases by the color in the metallographic photos after the special erosion. According to the chemical composition of the alloy and its crystallization characteristics, the gray orange region was confirmed to be the primary austenite phase and the deep gray and purple region was confirmed to be the eutectic austenite phase. The spheroidal carbide showed light orange color (the picric acid color) due to the good corrosion resistance. Also the spheroidal carbide can be confirmed to be the eutectic carbide according to the color differences.

During the metal mold casting process, the cooling rate of the liquid alloy was very high. The primary austenite dendrites nucleation rate increased and the dendrites grew rapidly when the temperature was below the liquidus line. The strong austenite dendrites crossed each other and the residual liquid phase was divided into a number of separate regions. Because the carbon content in the primary austenite was lower than the average carbon content in the alloy, more carbon would be rejected into the remaining melt during the cooling process. In this case, the carbon concentration in the residual liquid phase increased rapidly until it reached the eutectic composition. In addition, the high Si content promoted the formation of the spheroidal eutectic graphite in the alloy. Thus the austenite and spheroidal graphite eutectic microstructure were formed at the beginning of the eutectic stage. However, the cooling rate of the melt was very high, and the temperature was rapidly reduced during the solidification process. The austenite and carbide eutectic microstructure formed in the residual liquid phase regions following the non-equilibrium crystallization process when the temperature was far below the equilibrium system eutectic region ${ }^{[15]}$. The eutectic carbides were spheroidal carbides and the result was in good agreement with that reported by Jiang et al. ${ }^{[16]}$, which showed that the eutectic spheroidal carbide was very conducive to be formed in the isolated eutectic liquid phase region when the carbon concentration increased. The austenite dendrites grew preferentially along the opposite directions of the heat transfer ${ }^{[15]}$ and it made the isolated liquid phase region formed by the secondary dendrites intersected with each other have a specific direction. Therefore, the spheroidal carbide formed in the isolated liquid phase region might have certain orientation relationships with the austenite phase.

\subsection{Effect of carbon content on spheroidal carbide}

The distribution of the graphite and carbide in the ultra-low carbon ductile cast irons with different carbon contents is shown in Fig. 3. It can be seen that the amount of the spheroidal graphite and carbide increases with the increase of the carbon content. The diameter of the spheroidal carbides also increases with the increase of the carbon content and the distribution of the spherodial carbides is becoming uniform. It also can be seen that the volume fraction of the primary austenite dendrite decreases with the increase of the carbon content. However, the austenite dendrite is refined. The more developed secondary dendrite and the finer secondary austenite dendrite spacing in higher carbon content make the eutectic residual liquid divide into many isolated regions. Also it is very beneficial for the precipitation of the eutectic spheroidal carbide.

Carbon is the graphite and carbide formation element. Therefore, the amount of the graphite balls and carbides increases with the increase of the carbon content. Moreover, the constitutional supercooling of the solidification front of the austenite could happen with the increase of the carbon content. This is conducive to the growth of the austenite secondary dendrite. Then the space of the austenite dendrite became smaller and it would be easier for the eutectic liquid phase to be separated into many isolated regions. Therefore, it promoted the growth of the spheroidal carbide and made the spheroidal carbides distribution uniform.

\subsection{Nucleus of spheroidal carbide}

Figure 4 shows the morphology of the spheroidal carbides in the ultra-low carbon ductile cast iron. It can be seen that there is a small black spot in the middle of the spheroidal carbide. The composition of the small black spots was measured using EDS, and the corresponding quantitative analysis result is presented in Fig. 5. The analysis indicates that the spot $\mathrm{A}$ containing $\mathrm{Mg}$ and $\mathrm{O}$ is an oxide and the $\mathrm{Mg}$ and $\mathrm{O}$ stoichiometry ratio is approximately $1: 1$, as is seen in Figs. 5(a) and (b). The Mg element mainly came from the nodulizer during the spheroidizing process. The residual $\mathrm{Mg}$ and $\mathrm{O}$ in the liquid iron could generate the refractory $\mathrm{MgO}$ during the spheroidizing process. And it could serve as the nucleus of the eutectic spheroidal carbide due to its high melting point. Spot B mainly contained $\mathrm{C}, \mathrm{O}, \mathrm{Cr}, \mathrm{Fe}, \mathrm{Si}$ and $\mathrm{Mn}$ elements, as is seen in Figs. 5(c) and (d). Also it can be confirmed to be the oxide including $\mathrm{Cr}, \mathrm{Fe}, \mathrm{Si}, \mathrm{Mn}$ and other elements according to the EDS analysis.

The inoculant, modifier and nodulizer added to the ultra-low carbon ductile iron can promote the formation of the spherical graphite and improve its mechanical properties. The addition of the inoculant could provide the nucleus for the graphite balls ${ }^{[17,18]}$ and the nucleation substrates for the spheroidal carbides during the crystallization process. The temperature and the concentration fluctuations would arise around the inoculant 

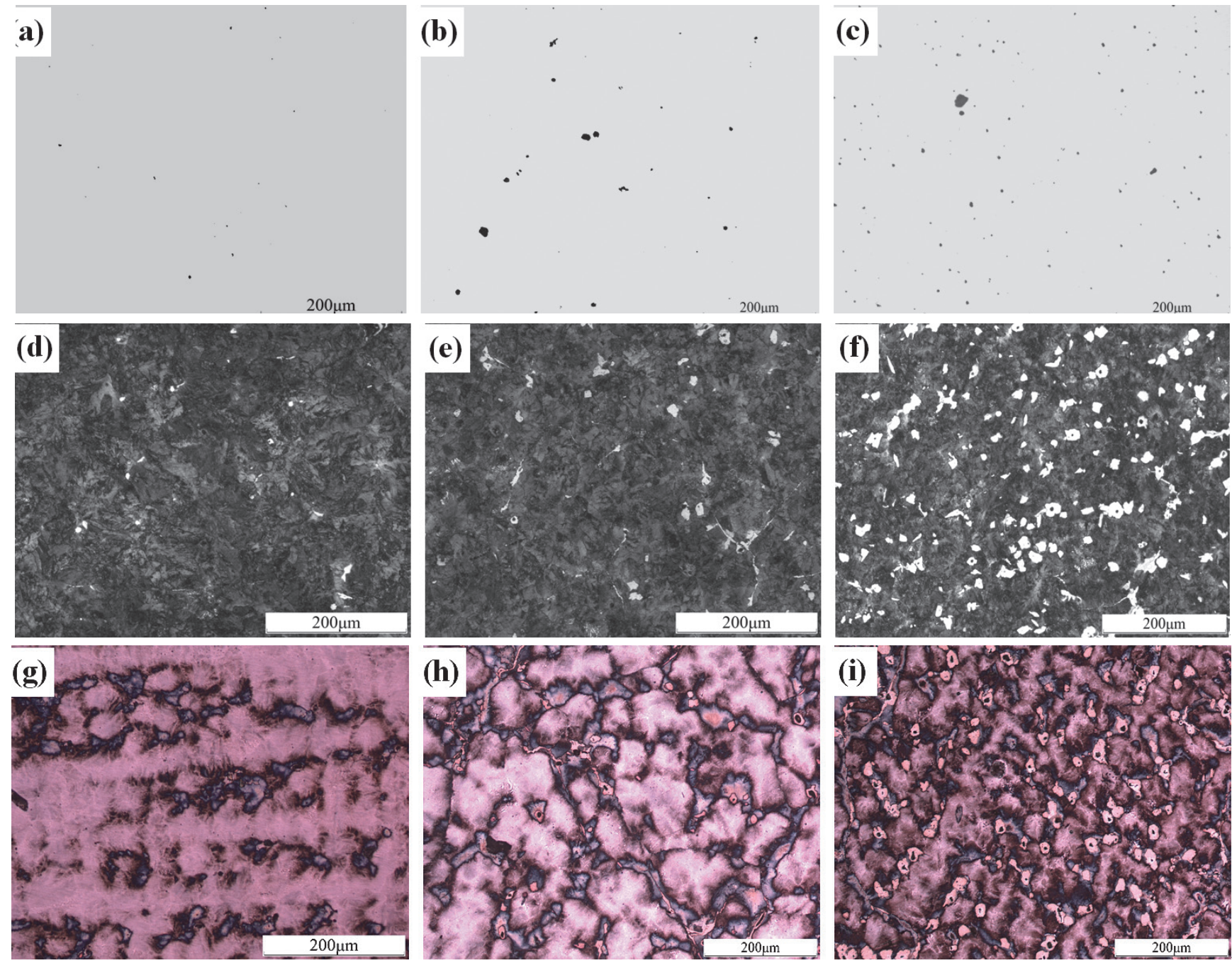

Fig. 3: Effect of carbon content on distribution of graphite and carbide with different metallographic techniques: (a), (d), (g) 0.79 wt.\% C; (b), (e), (h) 0.97 wt.\% C; (c), (f), (i) $1.20 w t . \%$ C and (a), (b), (c) unetched; (d), (e), (f) black and white; (g), (h), (i) color

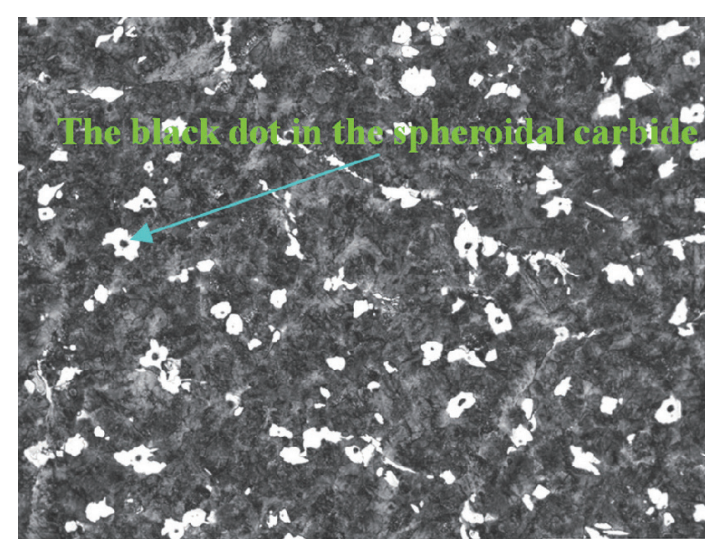

Fig. 4: Morphology of spheroidal carbides in ultralow carbon ductile cast iron

when the inoculant particles dissolved in the liquid irons. These particles and small oxide particles produced by the liquid iron treatment would be the nucleation core of the spheroidal carbide. According to the principle of the minimum free energy, the carbide eventually grows into spheroidal form. In addition, the constitutional supercooling could be caused by the addition of the modifier, and it was also good for the carbide attachment and the improvement of the roundness of the carbides ${ }^{[19]}$. Previous research has reported that the modifier would be helpful to improve the morphology of carbides ${ }^{[20]}$.

\subsection{Effect of cooling rate on the formation of spheroidal carbide}

The distribution of the graphite and carbide in different positions is shown in Fig. 6. It can be seen that the amount of the graphite balls gradually increases from the center to the edge of the specimen. However, the carbides are gradually changed from network and spheroid, a small number of networks and spheroids, to a perfect spherical. Also the distribution of the spheroidal carbide is more and more uniform.

The solidification time of the samples in R0-20 mm, R20$40 \mathrm{~mm}$, and R40-60 $\mathrm{mm}$ was $7.4 \mathrm{~min}, 3.7 \mathrm{~min}, 1.2 \mathrm{~min}$, respectively, which were calculated using numerical simulation technique. The cooling rate increased from the center to the edge. Therefore, the amount of the graphite balls gradually increased with an increase in the cooling rate ${ }^{[21-23]}$. The 

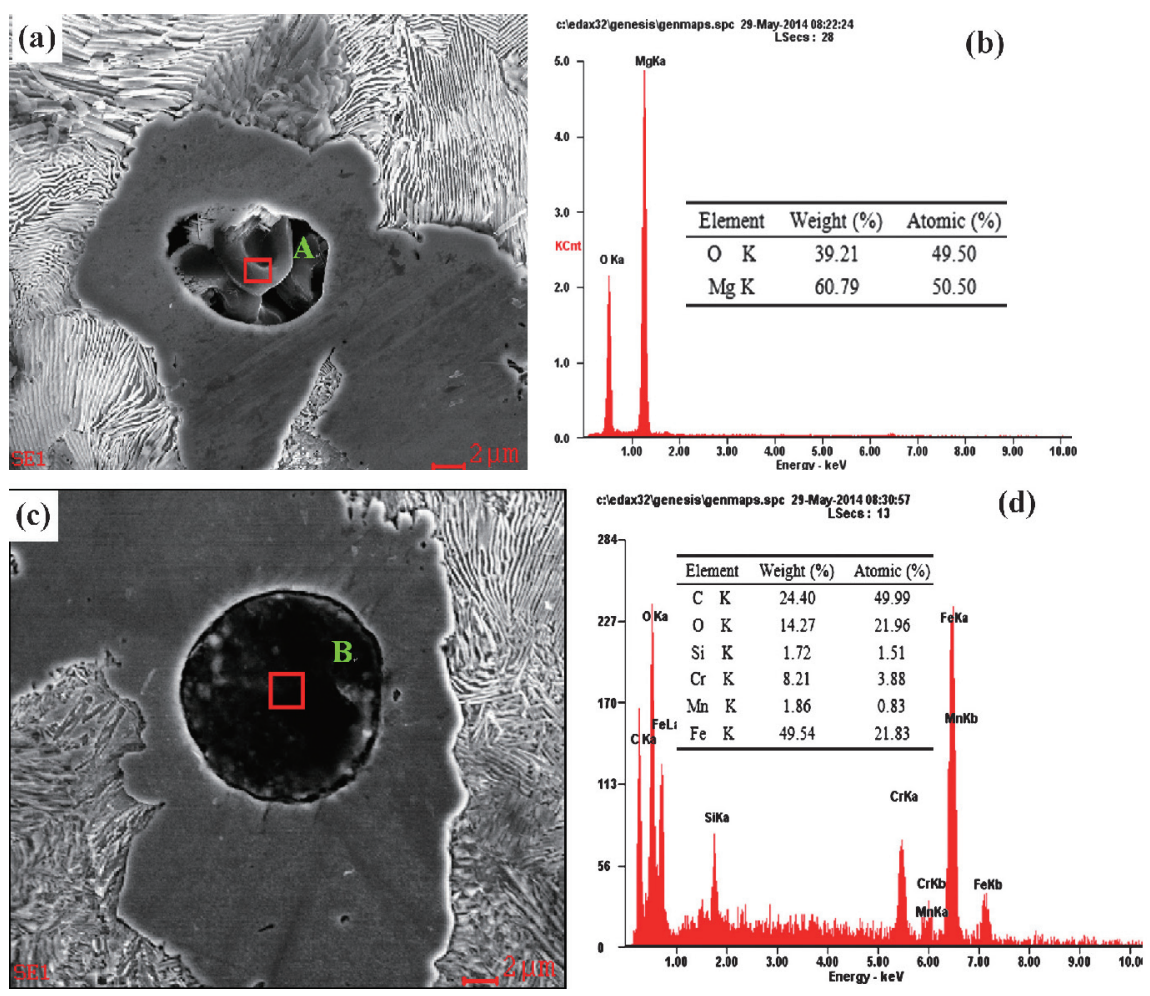

Fig. 5: (a) and (c) spheroidal carbide; (b), (d) EDS analysis results of black dot in spheroidal carbide in (a) and (c)
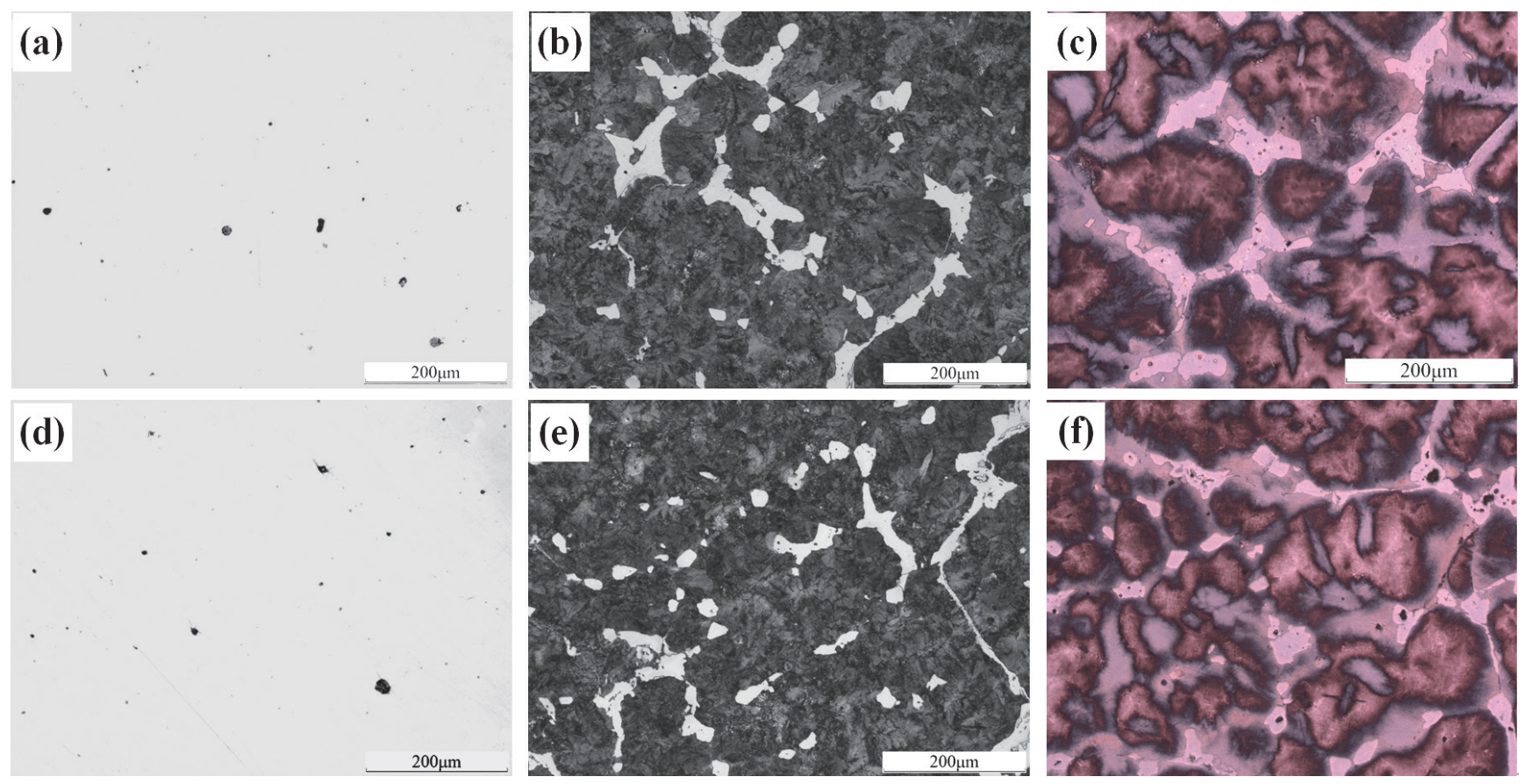

(g)
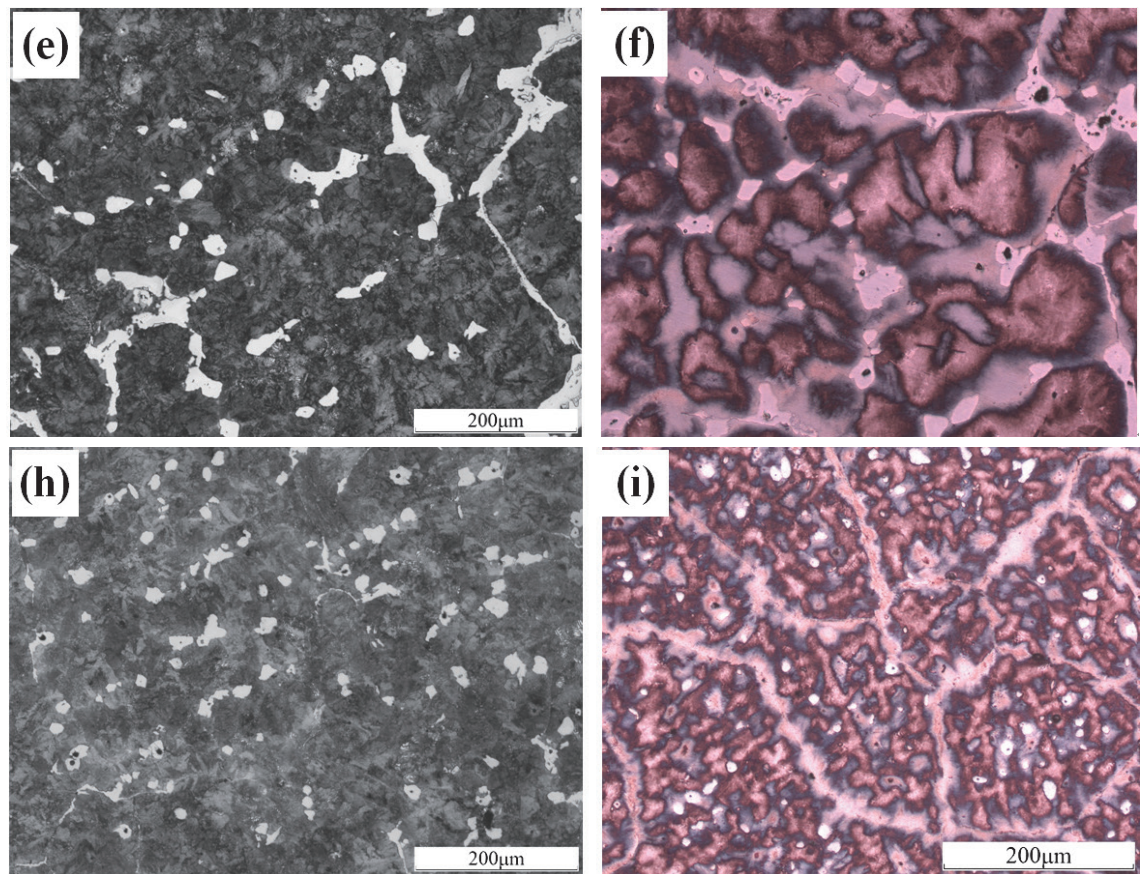

Fig. 6: Effect of cooling rate (different positions) on distribution of graphite and carbide used different metallographic techniques: (a), (b), (c) R0-20mm; (d), (e), (f) R20-40mm; (g), (h), (i) R40-60mm and (a), (d), (g) unetched; (b), (e), (h) black and white; (c), (f), (i) color 
morphology and quantity of the carbides were also related to the cooling rate. The cooling rate was slow in the center (R0$20 \mathrm{~mm}$ ) and the amount of the primary austenite dendrite crystal nucleus was less. So the nucleus had a long time to grow and it was easy to form the coarse dendrite. Thus, the isolated eutectic liquid phase regions would be expanded and some isolated areas would interconnect with each other. At the same time, the carbides would crystallize in the middle of the isolated liquid phase area. The network carbides would appear when some isolated carbides connected with each other. In this case, fewer spheroidal carbides and more network carbides would be obtained. When the cooling rate increased in the $1 / 2$ radius (R20-40 $\mathrm{mm}$ ) of the sample, the primary austenite dendrite crystal nucleus began to increase and the amount of the isolated eutectic liquid phase regions also increased. Then the spheroidal carbide would crystallize in the isolated area but the network carbide would be formed in the area where the residual liquid phase was not closed. Therefore, more spheroidal carbides and fewer network carbides would be obtained. The cooling rate was the fastest in the edge (R40-60 mm) of the sample. More primary austenite dendrite crystal nuclei and the faster growth rate made the austenite dendrite refined. Also the amount of the isolated eutectic liquid phase region became much greater. Accordingly, many more spheroidal carbides would be obtained and the distribution of the spheroidal carbides was homogeneous. As described above, the faster crystallization velocity was conducive to the formation of the spheroidal carbides and the lower crystallization velocity was beneficial to obtain the network carbides in a certain chemical composition.

\section{Conclusions}

(1) The eutectic graphite (equilibrium system) and spheroidal carbide (metastable system) can be obtained at the same time during the solidification process by controlling the chemical composition and cooling rate.

(2) A certain amount of carbon and cooling rate is a necessary condition for the formation of spherical graphites and spheroidal carbides. The amount of the spheroidal carbide increases with the increase of carbon content and cooling rate. The optimum carbon content was $1.20 \mathrm{wt} . \%$ and the best cooling rate was in the edge (R40-60 mm) of the sample.

(3) The carbides can be changed from network to spherical and the distribution of the spheroidal carbide was becoming uniform by modification treatment and the addition of heterogeneous nucleation.

\section{References}

[1] Panneerselvam S, Martis C J, Putatunda S K, et al. An investigation on the stability of austenite in Austempered Ductile Cast Iron (ADI). Materials Science and Engineering A, 2015, 626: 237-246.

[2] Cai Qizhou and Wei Bokang. Recent development of ductile cast iron production technology in China. China Foundry, 2008, 5(2): 82-91.

[3] Lin $\mathrm{C} \mathrm{K}$, Yang $\mathrm{C} \mathrm{H}$ and Wang $\mathrm{J} \mathrm{H}$. Corrosion fatigue of austempered ductile iron. Journal of Materials Science, 2003, 38 1667-1672.
[4] Chapetti M D. High-cycle fatigue of austempered ductile iron (ADI). International Journal of Fatigue, 2007, 29: 860-868.

[5] Kumari U R and Rao P P. Study of wear behaviour of austempered ductile iron. Journal of Materials Science, 2009, 44: 1082-1093.

[6] Benam A S, Yazdani S and Avishan B. Effect of shot peening process on fatigue behavior of an alloyed austempered ductile iron. China Foundry, 2011, 8(3): 325-330.

[7] Li Lingfang, Shu Xinfu, Li Yuzhong, et al. Mn+Cu alloyed bainite low carbon ductile iron. Modern Cast Iron, 2005(6): 14-16. (In Chinese)

[8] Xu Chunjie, Dai Pan, Zhang Zhengyang, et al. Effect of austempering parameters on microstructure and mechanical properties of horizontal continuous casting ductile iron dense bars. China Foundry, 2015, 12(2): 104-110.

[9] Benam A S. Effect of alloying elements on austempered ductile iron (ADI) properties and its process: Review. China Foundry, 2015, 12(1): 54-70.

[10] Gong Wenbang, Chen Guodong, Luo Li, et al. Design and control of chemical compositions for high-performance austempered ductile iron. China Foundry, 2012, 9(2): 143-147.

[11] Liu Yang. Process study on graphitic steel roller of suspended casting. Hot Working Technology, 2004(7): 43-44. (in chinese)

[12] Efremenko V G, Shimizu K, Cheiliakh A P, et al. Abrasive wear resistance of spheroidal vanadium carbide cast irons. Journal of Friction and Wear, 2013, 34(6): 466-474.

[13] Tomita Y. Effect of microstructure on tensile fracture of quenched and lightly tempered high carbon and low chromium steel containing undissolved spheroidal carbides. Journal of Materials Science, 1990, 25(11): 4745-4751.

[14] Sun Jun. Strength for decohesion of spheroidal carbide particlematrix interface. International Journal of Fracture, 1990, 44(4): R51-R56.

[15] Durand-Charre. Microstructure of steels and cast irons. Springer; Softcover reprint of hardcover 1st ed. 2004 (2010).

[16] Ding Hui, Gu Kechen, Liu Song, et al. Carbides in as-cast manganese steels. Journal of Shenyang University of Technology, 2002, 24(3): 185-188. (In chinese)

[17] Xiong Guoqing, Guo Xinqing, Yu Guomei, et al. Rare-earth inoculants for grey cast iron. Journal Huazhong University of Science and Technology, 1985, 13(1): 65-70. (In Chinese)

[18] Fras E, Gorny M. Inoculation effects of cast iron. Archives of Foundry Engineering, 2012, 4(12): 39-46.

[19] Hou Yuncheng, Wang You, Pan Zhaoyi, et al. Influence of rare earth nanoparticles and inoculants on performance and microstructure of high chromium cast iron. Journal of Rare Earths, 2012, 30(3): 283-288.

[20] Wu Xiaojun, Xing Jiandong, Fu Hanguang, et al. Development of refinement of the primary carbides in high chromium white cast iron. Foundry, 2006, 55(10): 999-1003. (In Chinese)

[21] Murcia S C, Ossa E A, and Celentano D J. Nodule evolution of ductile cast iron during solidification. Metallurgical and Materials Transactions B, 2014, 45(2): 707-718.

[22] Janowak J F and Gundlach R B. Approaching austempered ductile iron properties by controlled cooling in the foundry. Journal of Heat Treating, 1985, 4(1): 25-31.

[23] Gorny M and Tyrala E. Effect of cooling rate on microstructure and mechanical properties of thin-walled ductile iron castings. Journal of Materials Engineering and Performance, 2013, 22(1): 300-305. 